\title{
Estimativas de Tendência Genética para Características Produtivas em um Rebanho do Ecótipo Mantiqueira ${ }^{1}$
}

\section{Marcos Vinícius Gualberto Barbosa da Silva ${ }^{2,3}$, William José Ferreira ${ }^{3}$, Jaime Araujo Cobuci ${ }^{3}$, Guilherme Paes Guaragna², Paulo Rogério Palma de Oliveira ${ }^{2}$}

\begin{abstract}
RESUMO - Os registros utilizados neste estudo foram provenientes das cinco primeiras lactações de 1406 vacas do ecótipo Mantiqueira, filhas de 113 reprodutores, com partos entre os anos de 1952 e 1997. As informações sobre as características produção total de leite (PL), produção de gordura (PG), duração da lactação (DL) e período seco (PS) foram obtidas do NPZGJRA/IZ/APTA/SAA. Com base nas predições dos valores genéticos, para cada característica estudada, estimaram-se os diferenciais de seleção e as tendências genéticas, por meio de quatro trajetórias de seleção: touros pais de touros (SB), vacas mães de touros (DB), touros pais de vacas (SC) e vacas mães de vacas (DC). Os períodos estudados compreenderam os anos de 1952 a 1976, primeiro período, que representou a formação e a ampliação do rebanho; de 1977 a 1997, segundo período, caracterizado pela implementação de um programa de melhoramento genético do ecótipo; e de 1952 a 1997, período total. As análises foram feitas por meio do sistema MTDFREML, com um modelo animal que incluiu os efeitos fixos de ano-estação de parto e duração da lactação e, como covariável, a idade da vaca no parto, em meses, com termos linear e quadrático. Como efeitos aleatórios foram incluídos animal, efeito permanente de meio e erro. Verificou-se que os diferenciais de seleção das trajetórias SB e SC foram superiores aos das demais trajetórias, no primeiro período, enquanto no segundo período, no entanto, em razão da mudança de critério de seleção, os diferenciais de seleção das trajetórias DB e DC foram maiores. As tendências genéticas obtidas para PG, DL e PS foram inexpressivas, o que sugere que a seleção tenha sido direcionada somente para PL. As taxas de ganho genético podem ser aumentadas, razão pela qual o programa de melhoramento deve ser reestruturado.
\end{abstract}

Palavras-chave: bovino de leite, diferencial de seleção, ecótipo Mantiqueira, método REML, modelo animal, tendência genética

\section{Genetic Trend Estimates for Yield Traits in Mantiqueira Cattle}

ABSTRACT - The data used in this study are from the first five lactations of 1,406 cows of the Mantiqueira Ecotype, daughters of 113 sires, with calving from 1952 to 1997. The total milk yield (PL), fat production (PG), lactation period (DL), and days dry (PS) were obtained from NPZGJRA/IZ/APTA/SAA. Based on the genetic values predictions, for each studied feature selection differentials and genetic trends were estimated though four selection paths of selection: bulls to breed bulls (SB), cows to breed bulls (DB), bulls to breed cows (SC) and cows to breed cows (DC). Two periods were involved: the first, from 1952 to 1976, representing the formation period and herd increase, the second, from 1977 to 1997, when the breeding program was implemented. The whole period from 1952 to 1997 was also considered. MTDFREML was used in the analysis with an animal model containing the year-season effects of calving, lactation period, the linear and quadratic effects of age of calving as fixed effects. Animal and permanent environment and error were included as random effects. The selection differentials for the SB and SC paths were larger in the first period. DB and DC paths were larger in the second period due to changes in the selection criterion. Selected gains for PG, DL, and PS paths were very small suggesting that the selection was only for PL. Rates of gains could be increased with changes in the breeding program.

Key Words: animal model, dairy cattle, genetic trend, Mantiqueira ecotype, REML methodology, selection differential

\section{Introdução}

Em 1952, o antigo Departamento da Produção Animal, hoje Instituto de Zootecnia, da Secretaria de Agricultura e Abastecimento do Estado de São Paulo, adquiriu uma amostra de bovinos malhados que tinham como ascendentes a raça Holandesa e o gado Crioulo da região (GUARAGNA et al., 1984). Até
1976, a criação desses animais objetivava ampliar o rebanho e nenhum trabalho visando à avaliação destes foi realizado.

O programa de melhoramento genético do ecótipo Mantiqueira foi iniciado em 1977, no Núcleo de Pesquisas Zootécnicas Geraldo José Rodrigues Alckmin, do Instituto de Zootecnia da Agência Paulista de Tecnologia dos Agronegócios da Secretaria de

\footnotetext{
1 INSTITUTO DE ZOOTECNIA - Parte integrate do projeto IZ - 14-001/52.

2 Pesquisador Científico - Núcleo de Pesquisa Zootécnica Geraldo J.R. Alckmin/IZ/APTA/SAA CP 07 - Pindamonhagaba - SP.

3 Aluno de Pós-graduação, DZO/UFV. E.mail: mbarbosa@tdnet.com.br; wjferreira@tdnet.com.br; ds35625@correio.cpd.ufv.br
} 
Agricultura e Abastecimento do Estado de São Paulo (NPZGJRA/IZ/APTA/SAA-SP), no município de Pindamonhangaba, Estado de São Paulo, com o intuito tornar disponível aos produtores do Vale do Paraíba paulista um grupamento genético que apresentasse bons desempenhos produtivos e reprodutivos, sob as condições de criação da região (GUARAGNA et al., 1988).

Como em qualquer programa, faz-se necessário acompanhar os resultados para que se possa avaliar sua evolução, bem como efetuar ajustes, de modo eficiente, com vistas na otimização do ganho genético e no aumento da rentabilidade da exploração. Uma das maneiras de se realizar esse monitoramento é por meio da avaliação da tendência genética ao longo do tempo, pela qual se avalia a mudança ocasionada pelo processo de seleção. Avaliações de tendências genéticas em diferentes raças têm sido objeto de estudo de vários pesquisadores, tanto em rebanhos experimentais (SANTOS et al., 1990) quanto em rebanhos comerciais (BASTOS et al., 1998).

As características produtivas (produção de leite, gordura e proteína) são quantitativas e refletem os efeitos combinados de grande número de genes, sendo também significativamente influenciadas por fatores de ambiente (NIZAMANI e BERGER, 1996). Essas características são as de maior valor econômico, razão pela qual são normalmente consideradas como essenciais em programas de seleção (ROMAN et al., 1999).

RENDEL e ROBERTSON (1950) relataram que são possíveis ganhos genéticos anuais, para produção de leite, superiores a $2 \%$ da média. Para isso é necessária intensa e precisa seleção dos animais a serem utilizados como pais de touros e de vacas, bem como redução do intervalo de gerações. VAN TASSELL e VAN VLECK (1991) destacaram que as causas de ganhos inferiores a $2 \%$ podem ser a ênfase em outras características, e não na produção de leite; o tratamento preferencial; a baixa intensidade de seleção de touros pais de touros; e os longos intervalos de geração para touros e vacas, selecionados como pais de touros.

Neste trabalho, objetivou-se estimar os diferenciais de seleção e as tendências genéticas para algumas características produtivas, por meio de quatro trajetórias de seleção, propostas por RENDEL e ROBERTSON (1950), quais sejam, touros pais de touros (SB), vacas mães de touros (DB), touros pais de vacas (SC) e vacas mães de vacas (DC).

\section{Material e Métodos}

\section{Dados e modelos}

Os registros utilizados neste estudo foram provenientes das cinco primeiras lactações de 1.406 vacas do ecótipo Mantiqueira, filhas de 113 reprodutores, cujos partos compreenderam os anos de 1952 a 1997. As informações sobre as características produção total de leite (PL), produção de gordura (PG), duração da lactação (DL) e período seco (PS) foram obtidas do Arquivo de Escrituração Zootécnica do NPZGJRA/IZ/APTA/SAA-SP. Este rebanho, cuja formação foi iniciada em 1952, a partir da compra de 50 animais (entre machos e fêmeas) de algumas propriedades de Minas Gerais e São Paulo, foi manejado até 1974, com vista, basicamente na ampliação do rebanho. Neste ano, verificou-se, então, a necessidade de se avaliar o material genético existente, sendo montada uma unidade experimental de seleção, cuja principal característica era a predominância do uso de pastagem na alimentação (GUARAGNA et al., 1984). Para isso, foi elaborado um plano de acasalamento de animais não-aparentados, cujo critério considerado era a não-existência de avós em comum, e foram também selecionados quatro touros de origens distintas ("linhagens"), para serem os pais do primeiro grupo de progênie. Neste grupo, em que o primeiro parto ocorreu no ano de 1977 , iniciou-se o programa de avaliação do material genético, sendo em 1984, formadas mais seis "linhagens".

A partir de 1977, ocorreram mudanças no manejo, como, por exemplo, passagem da ordenha manual à mecânica e criação artificial de bezerros. Todavia, o sistema de produção era direcionado para a criação dos animais a pasto. Até este ano, as vacas eram, aparentemente, selecionadas com base na produção de leite, sem qualquer tipo de correção para efeitos não-genéticos. Os touros eram escolhidos de acordo com o desempenho produtivo de suas mães, isto é, eram selecionados aqueles reprodutores machos cujas mães apresentassem maior produção de leite.

A partir do início do programa de melhoramento, em 1977, procurou-se ter um manejo definido e uniforme na Unidade Experimental de Seleção do Tipo Mantiqueira, em que a principal característica era a predominância do uso de pastagem na alimentação, formada, basicamente, pelos capins Brachiaria decumbens, Setaria kazungula e Mellinis minutiflora. Na época da seca (abril a setembro), era fornecida aos animais uma suplementação vo- 
lumosa, como silagem de milho ou sorgo. As vacas em lactação eram ordenhadas mecanicamente, sem bezerro ao pé, duas vezes ao dia, e recebiam suplementação concentrada, na base de $1 \mathrm{~kg}$ de ração para cada $2,5 \mathrm{~kg}$ de leite produzido, acima de $3 \mathrm{~kg}$.

Durante o programa de melhoramento, todas as novilhas eram incorporadas ao rebanho, desde que não apresentassem problemas reprodutivos ou defeitos anatômicos, e disponibilizadas para reprodução aos dois anos de idade ou quando atingissem peso vivo em torno de $320 \mathrm{~kg}$ (GUARAGNA et al., 1988).

Após encerrarem a segunda lactação, as vacas eram classificadas em três categorias, quais sejam, Elite, Média (ou Comercial) e Fundo, de acordo com os critérios existentes na Tabela 1. Para que um animal fosse considerado da Elite, ele teria de apresentar pelagem e cor de cascos classificados como típicos e, pelo menos, outros dois quesitos bons e um médio. Para ser classificado como Fundo, a produção e a fertilidade teriam de ser consideradas como fracas ou existir caracteres genéticos indesejáveis, sendo as demais consideradas como Médias ou Comerciais.

Quanto aos machos, eram selecionados, aproximadamente, dez por ano para serem usados como reprodutores, com base na primeira lactação de suas mães, no desenvolvimento ponderal e no tipo (pelagem e cor de cascos, classificados como típicos); a partir de 1984, procurou-se selecionar apenas um touro de cada linhagem. A fertilidade desses animais (mensuração testicular, teste de libido, características seminais etc) era avaliada e, aproximadamente, aos três anos de idade eram disponibilizados para reprodução.

Os períodos de estudo compreenderam os anos de 1952 a 1976, nos quais foi o rebanho formado e ampliado; de 1977 a 1997, caracterizado pela implementação do programa de melhoramento genético do ecótipo; de 1952 a 1997, período total.

Para consistência do arquivo analisado, exigiu-se que cada subclasse ano-estação contivesse, no mínimo, 15 observações e que cada reprodutor tivesse

Tabela 1 - Critérios para classificação das vacas Mantiqueira Table 1 - Approaches for classification of the Mantiqueira cows

\begin{tabular}{|c|c|c|}
\hline $\begin{array}{l}\text { Característica } \\
\text { Trait }\end{array}$ & $\begin{array}{c}\text { Classe } \\
\text { Class }\end{array}$ & $\begin{array}{c}\text { Critérios } \\
\text { Approaches }\end{array}$ \\
\hline Pelagem & Típica & Preta e tribofe \\
\hline Coat & $\begin{array}{l}\text { Typical } \\
\text { Não típica }\end{array}$ & $\begin{array}{l}\text { Black or tribofe } \\
\text { Vermelha } \\
\text { Red }\end{array}$ \\
\hline Cor dos cascos & Típica & Todos os cascos pretos \\
\hline Hoofs color & $\begin{array}{l}\text { Typical } \\
\text { Não típica } \\
\text { Not typical }\end{array}$ & $\begin{array}{l}\text { All black hoofs } \\
\text { Pelo menos um casco branco } \\
\text { At least a white hoof }\end{array}$ \\
\hline Produção de leite ( $1^{\mathrm{a}}$ lactação) & Fraca & $\leq 1.980 \mathrm{~L}(300$ dias $)$ \\
\hline Milk yield ( $1^{\text {st }}$ lactation $)$ & $\begin{array}{l}\text { Weak } \\
\text { Média } \\
\text { Mean } \\
\text { Boa } \\
\text { Good }\end{array}$ & $\begin{array}{l}\leq 1,980 \mathrm{~L}(300 \text { days }) \\
1980 \mathrm{~L} \text { a } 2880 \mathrm{~L}(300 \text { dias }) \\
1980 \mathrm{~L} \text { to } 2880 \mathrm{~L}(300 \text { days }) \\
\text { acima de } 2880 \mathrm{~L} \\
\text { above of } 2880 \mathrm{~L}\end{array}$ \\
\hline Fertilidade $^{1}$ & Fraca & $\mathrm{IPP}^{2} \geq 4$ anos e $\mathrm{IDP}^{3}>18 \mathrm{~m}$ \\
\hline Fertility $^{l}$ & $\begin{array}{l}\text { Weak } \\
\text { Média } \\
\text { Mean } \\
\text { Boa } \\
\text { Good }\end{array}$ & $\begin{array}{l}I P P^{2} \geq 4 \text { years e IDP }>18 \mathrm{~m} \\
\text { IPP de } 3 \text { a } 4 \text { anos e IDP de } 14 \text { a } 18 \mathrm{~m} \\
I P P \text { of } 3 \text { to } 4 \text { years and IDP of } 14 \text { to } 18 \mathrm{~m} \\
\text { IPP }<3 \text { anos e IDP }<18 \mathrm{~m} \\
\text { IPP }<3 \text { years and IDP }<18 \mathrm{~m}\end{array}$ \\
\hline Tipo $^{4}$ & Fraca & 50 pontos \\
\hline Type 4 & $\begin{array}{l}\text { Weak } \\
\text { Média } \\
\text { Mean } \\
\text { Boa } \\
\text { Good }\end{array}$ & $\begin{array}{l}50 \text { points } \\
51 \text { a } 70 \text { pontos } \\
51 \text { to } 70 \text { points } \\
\text { acima de } 70 \text { pontos } \\
\text { above of } 70 \text { points }\end{array}$ \\
\hline
\end{tabular}

${ }_{1}^{1}$ Avaliada após o primeiro parto (Evaluated after the first calving).

2 IPP = Idade ao primeiro parto (Age of first calving).

3 IDP = Intervalo de partos (Calving interval).

${ }^{4}$ Aparência geral - 30 pontos (Appearance general - 30 points), caracterização leiteira - 20 pontos (angularity - 20 points), conformação - 20 pontos (frame - 20 points) e sistema mamário - 30 pontos (and udder - 30 points) (Adaptado de GUARAGNA et al., 1984) [Adapted from GUARAGNA et al., 1984]. 
filhas em, pelo menos, dois anos. Foi utilizado um arquivo de pedigree que continha 2070 animais diferentes, com todas as informações de parentesco disponíveis, desde a formação do rebanho em 1952. $\mathrm{O}$ coeficiente de endogamia médio do rebanho foi igual a 5,8\%; mínimo, de 1,0\%, e máximo, de 27,0\%.

As análises de predição dos valores genéticos, para cada característica, foram efetuadas pelo sistema MTDFREML (BOLDMAN et al., 1995), em que em um modelo animal se incluíram os efeitos fixos de ano-estação de parto (águas - outubro a março, e seca - abril a setembro); como covariável, a idade da vaca no parto, em meses, com termos linear e quadrático, além dos efeitos aleatórios de animal, de ambiente permanente e erro. Nas análises das produções de leite e de gordura, incluiu-se, ainda, o efeito fixo da duração da lactação.

Cálculo do diferencial de seleção

As médias dos valores genéticos foram calculadas por ano de nascimento dos animais, em cada trajetória de seleção: touros pais de touros (SB), touros pais de vacas (SC), vacas mães de touros (DB) e vacas mães de vacas (DC). Para touros, foram calculadas duas médias por trajetória de seleção - ponderada pelo número de progênies e não-ponderada, enquanto para as vacas foram calculadas somente as médias nãoponderadas. As médias ponderadas foram interpretadas como medida representativa do mérito de touros, usados como os pais da próxima geração, enquanto as médias não-ponderadas forneceram a estimativa do mérito genético dos touros ou das vacas, que foram avaliados ao longo dos anos.

Os diferenciais de seleção foram calculados, por ano de nascimento, como a diferença entre as médias dos valores genéticos em cada trajetória de seleção e a média dos valores genéticos de todas as vacas nascidas durante o mesmo período. Todas as vacas nascidas durante determinado ano foram usadas como base de comparação para os cálculos dos diferenciais de seleção, uma vez que as vacas não eram selecionadas.

\section{Cálculo do ganho genético}

O ganho genético realizado para cada trajetória de seleção foi estimado por meio de regressão das médias dos valores genéticos, para as características estudadas, sobre o ano de nascimento. As médias ponderadas e não-ponderadas foram usadas para as trajetórias SB e SD, com o intuito de considerar as diferenças do número de progênies de cada touro.
Ganhos genéticos foram estimados para o primeiro período (1952 a 1976), para o segundo (1977 a 1997) e para todo o período (1952 a 1997).

Os ganhos genéticos anuais $(\Delta \mathrm{g})$, para as características estudadas, foram calculados pelos os coeficientes de regressão dos valores genéticos sobre o ano de nascimento $(\mathrm{T})$, para cada trajetória de seleção $\left(b_{\mathrm{G}}\right)$, conforme proposto por VAN TASSELL e VAN VLECK (1991):

$$
\Delta \mathrm{g}=1 / 4\left(\mathrm{~b}_{\mathrm{G}_{\mathrm{SB}} \cdot \mathrm{T}}+\mathrm{b}_{\mathrm{G}_{\mathrm{DB}} \cdot \mathrm{T}}+\mathrm{b}_{\mathrm{G}_{\mathrm{SC}} \cdot \mathrm{T}}+\mathrm{b}_{\mathrm{G}_{\mathrm{DC}} \cdot \mathrm{T}}\right) .
$$

Os erros-padrão, associados às estimativas de ganho genético anual, foram obtidos por meio da média dos erros-padrão dos coeficientes de regressão de cada trajetória, conforme sugerido por NIZAMANI e BERGER (1996).

\section{Resultados e Discussão}

\section{Diferenciais de seleção}

Médias dos valores genéticos dos animais de diferentes trajetórias, por ano de nascimento, para produção total de leite são apresentadas nas Figuras $1,2,3$ e 4 . Os valores genéticos médios do grupo base não-selecionado, isto é, todas as vacas nascidas durante o mesmo ano, estão também incluídos em cada figura, de modo a possibilitar a comparação das tendências. Os diferenciais de seleção anuais para cada trajetória de seleção são obtidos pelas diferenças entre as médias dos valores genéticos dos pais, ponderadas ou não-ponderadas, e as médias dos valores genéticos do grupo base não-selecionado.

\section{Touros pais de touros (SB)}

As médias anuais dos valores genéticos para SB são mostradas na Figura 1. No primeiro período, de 1952 a 1976, o diferencial de seleção para produção de leite aumentou após 1964. A partir de 1977, dada a mudança nos critérios de seleção, em decorrência do início do programa de melhoramento, houve declínio no diferencial de seleção desta trajetória, que só passou a ser positivo após 1988. De modo geral, em todo o período avaliado, as médias ponderadas e não-ponderadas foram semelhantes, o que pode ser explicado pelo fato de quase todos os reprodutores terem deixado filhos, que foram utilizados como touros no rebanho. Os diferenciais de seleção obtidos nesta trajetória não seguiram a mesma tendência dos apresentados por VAN TASSEL e VAN VLECK (1991), na raça Holandesa, e por 


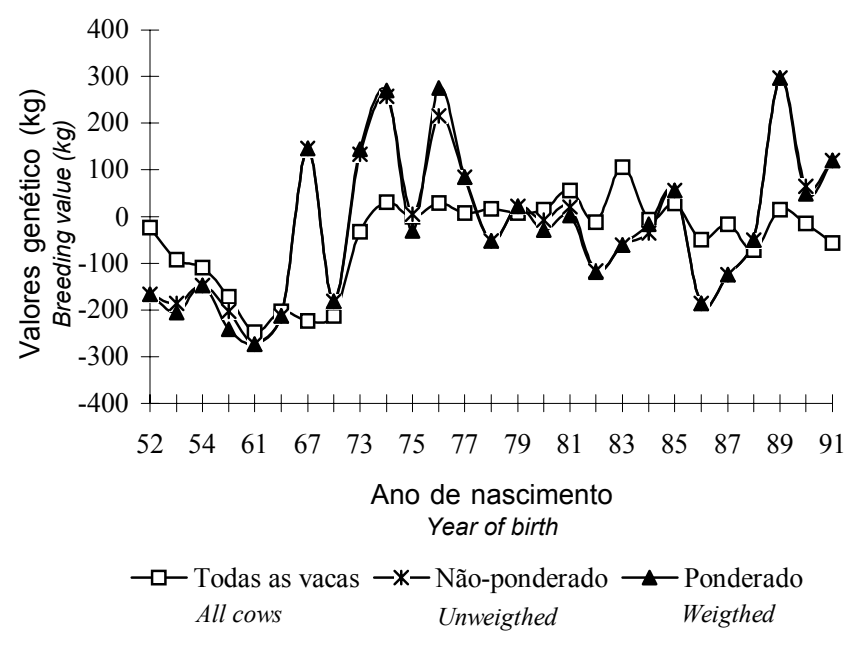

Figura 1 - Médias dos valores genéticos para produção de leite dos touros pais de touros, ponderados pelo número de filhos e não-ponderados, de acordo com o ano de nascimento dos touros, comparadas com as médias dos valores genéticos de todas as vacas nascidas durante 0 mesmo ano.

Figure 1 - Means of breeding value for milk yield for sires of bulls weighted by number of sons and unweighted by year of birth of sires compared with the means of breeding value of all cows born during the same year.

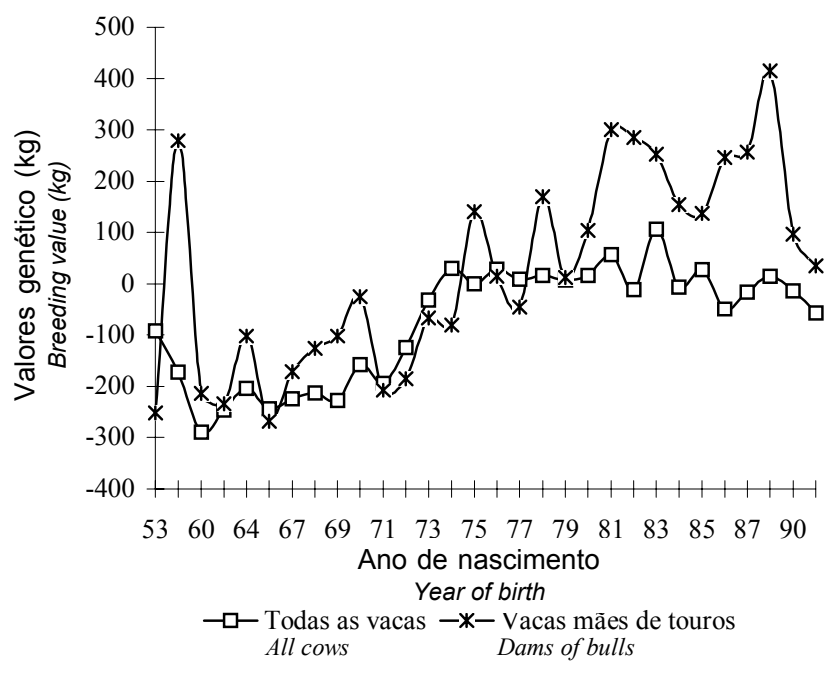

Figura 2 - Médias dos valores genéticos para produção de leite das vacas mães de touros de acordo com o ano de nascimento das vacas, comparadas com as médias dos valores genéticos de todas as vacas nascidas durante o mesmo ano.

Figure 2 - Means of breeding value for milk yield for dams of bulls by year of birth of dams compared with the means of breeding value of all cows born during the same year.

a seleção de melhores vacas para serem mães de reprodutores. Observa-se também (Figura 5), que no segundo período, a seleção de vacas que seriam mães de touros foi mais eficiente do que a de reprodutores.

Na Tabela 2, pode-se observar que o diferencial de seleção médio para produção de leite foi, aproximadamente, três vezes maior no segundo período do que no primeiro. Destaca-se ainda que o diferencial de seleção desta trajetória foi superior aos obtidos das demais. Para as outras características estudadas, os diferenciais de seleção médios, no segundo período, foram menores.

\section{Touros pais de vacas (SC)}

As médias anuais dos valores genéticos para produção de leite, na trajetória $\mathrm{SC}$, são apresentadas na Figura 3. A seleção para produção de leite foi mais intensa nos anos de 1966 a 1977, nos quais foram obtidos os maiores diferenciais. A partir de 1977, o diferencial de seleção diminuiu, bem como a média dos valores genéticos dos touros pais de vacas. Verifica-se que, após o ano de 1988, houve aumentos nos diferenciais de seleção e nos valores genéticos.

As respostas obtidas por seleção, determinadas pelas médias não-ponderadas dos valores genéticos 


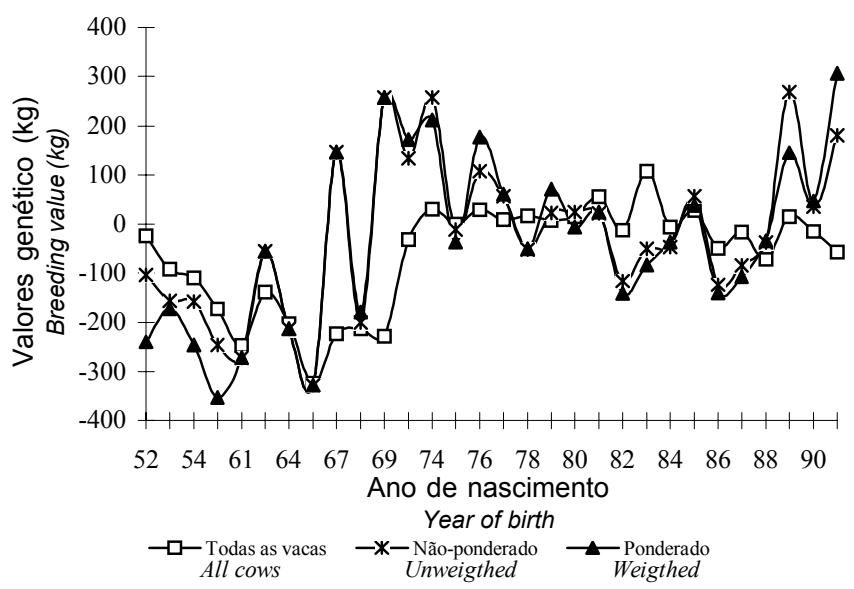

Figura 3 - Médias dos valores genéticos para produção de leite dos touros pais de vacas, ponderados pelo número de filhas e não-ponderados, de acordo com o ano de nascimento dos touros, comparadas com as médias dos valores genéticos de todas as vacas nascidas durante o mesmo ano.

Figure 3 - Means of breeding value for milk yield for sires of cows weighted by number of daughters and unweighted by year of birth of sires compared with the means of breeding value of all cows born during the same year.

De modo análogo ao observado na trajetória de touros pais de touros (SB), a seleção de touros pais de vacas foi mais eficiente no primeiro período (1952 a 1976) do que no segundo (a partir de 1977). As demais características estudadas tiveram comportamento semelhante à produção de leite.

Vacas mães de vacas (DC)

As médias anuais dos valores genéticos para produção de leite, nesta trajetória de seleção, são apresentadas na Figura 4. A seleção de DC foi menos intensa do que para as demais trajetórias, como esperado, devido à baixa taxa reprodutiva das vacas e à intensidade de seleção praticada. Ao longo do programa de melhoramento, segundo período, o descarte de vacas somente era realizado em decorrência de doenças ou de injúrias que comprometessem a produção. Desse modo, houve aumento no número de vacas, o que tornou disponível mais animais para reprodução. Nesse período, observou-se ainda aumento nos diferenciais de seleção médios (Tabela 2), dada a maior eficiência na seleção de vacas para serem mães de vacas.

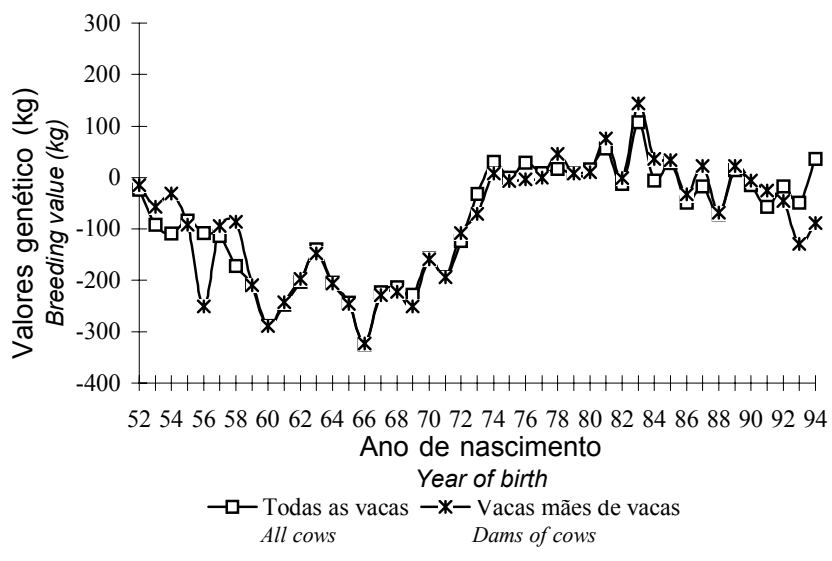

Figura 4 - Médias dos valores genéticos para produção de leite das vacas mães de vacas de acordo com o ano de nascimento de vacas, comparadas com as médias dos valores genéticos de todas as vacas nascidas durante o mesmo ano.

Figure 4 - Means of breeding value for milk yield for dams of cows by year of birth of dams compared with the means of breeding value of all cows born during the same year.

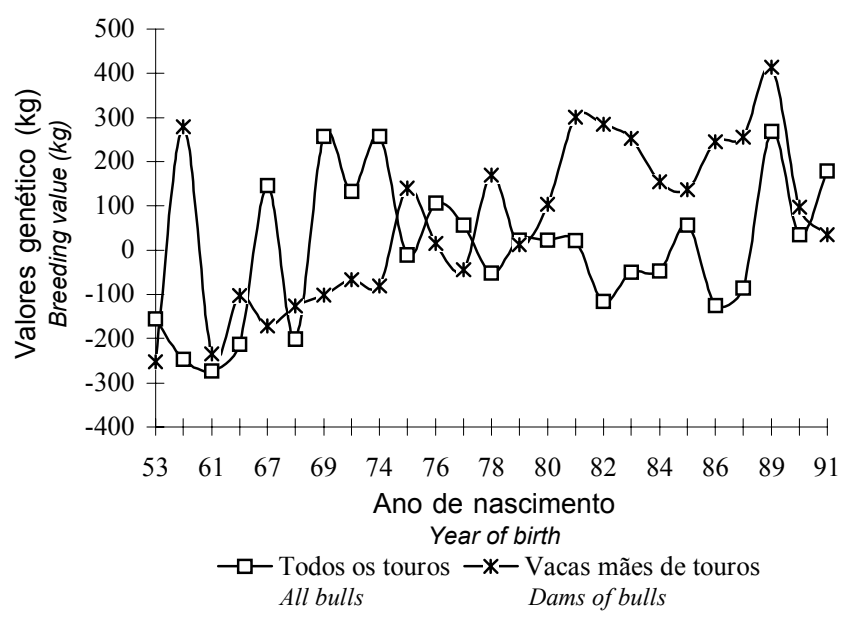

Figura 5 - Médias dos valores genéticos para produção de leite dos touros e vacas mães de touros, ao longo dos anos de nascimento.

Figure 5 - Means of breeding value for milk yield for sires and dams of sires, by year of birth. 
Tabela 2 - Estimativas do diferencial de seleção médio para produção de leite, produção de gordura, duração da lactação e período seco, para cada trajetória de seleção Table 2 - Estimated average selection differential for milk yield, fat yield, lactation length and days dry, for each path of selection

\begin{tabular}{|c|c|c|c|c|c|c|c|c|c|c|c|c|}
\hline \multirow[b]{2}{*}{$\begin{array}{l}\text { Trajetória de } \\
\text { Seleção }^{1} \\
\text { Selection path }^{1}\end{array}$} & \multicolumn{4}{|c|}{$\begin{array}{c}1^{\circ} \text { período }(1952-1976) \\
1^{s t} \text { period }\end{array}$} & \multicolumn{4}{|c|}{$\begin{array}{c}2^{\circ} \text { período }(1977-1997) \\
2^{\text {nd }} \text { period }\end{array}$} & \multicolumn{4}{|c|}{$\begin{array}{c}\text { Período total }(1952-1997) \\
\text { Whole period }\end{array}$} \\
\hline & $\begin{array}{l}\text { Leite } \\
\text { Milk }\end{array}$ & $\begin{array}{c}\text { Gordura } \\
\text { Fat }\end{array}$ & $\begin{array}{c}\text { Duração da } \\
\text { lactação } \\
\text { Lactation } \\
\text { lenght }\end{array}$ & $\begin{array}{c}\text { Período } \\
\text { seco } \\
\text { Days } \\
\text { dry }\end{array}$ & $\begin{array}{l}\text { Leite } \\
\text { Milk }\end{array}$ & $\begin{array}{l}\text { Gordura } \\
\text { Fat }\end{array}$ & $\begin{array}{c}\text { Duração da } \\
\text { lactação } \\
\text { Lactation } \\
\text { lenght }\end{array}$ & $\begin{array}{c}\text { Período } \\
\text { seco } \\
\text { Days } \\
\text { dry }\end{array}$ & $\begin{array}{l}\text { Leite } \\
\text { Milk }\end{array}$ & $\begin{array}{c}\text { Gordura } \\
\text { Fat }\end{array}$ & $\begin{array}{c}\text { Duração da } \\
\text { lactação } \\
\text { Lactation } \\
\text { lenght }\end{array}$ & $\begin{array}{c}\text { Período } \\
\text { seco } \\
\text { Days } \\
\text { dry }\end{array}$ \\
\hline$\overline{\mathrm{SB}}$ & & & & & & & & & & & & \\
\hline $\begin{array}{l}\text { Ponderado } \\
\text { Weighed }\end{array}$ & 137,00 & 1,60 & 16,36 & $-17,11$ & $-7,17$ & 0,30 & 1,71 & $-2,17$ & 30,69 & 0,60 & 5,68 & $-6,05$ \\
\hline $\begin{array}{l}\text { Não-ponderado } \\
\text { Unweighed }\end{array}$ & 101,10 & 1,22 & 14,36 & $-14,38$ & $-5,56$ & 0,35 & 1,86 & $-2,75$ & 20,25 & 0,54 & 4,98 & $-5,57$ \\
\hline DB & & & & & & & & & & & & \\
\hline $\begin{array}{l}\text { Não-ponderado } \\
\text { Unweighed } \\
\text { SC }\end{array}$ & 50,74 & 1,40 & 9,09 & $-6,52$ & 176,37 & 0,19 & 7,40 & $-13,94$ & 87,77 & 0,42 & 5,61 & $-7,34$ \\
\hline $\begin{array}{l}\text { Ponderado } \\
\text { Weighed }\end{array}$ & 99,72 & 0,96 & 7,46 & $-11,31$ & $-21,99$ & 0,39 & 0,73 & $-0,14$ & 8,02 & 0,43 & 1,91 & $-2,69$ \\
\hline $\begin{array}{l}\text { Não-ponderado } \\
\text { Unweighed } \\
\text { DC }\end{array}$ & 64,44 & 0,66 & 7,68 & $-8,38$ & 2,60 & 0,35 & 1,83 & $-1,55$ & 11,87 & 0,31 & 2,74 & $-2,61$ \\
\hline $\begin{array}{l}\text { Não-ponderado } \\
\text { Unweighed }\end{array}$ & $-9,15$ & $-0,07$ & $-0,05$ & 0,24 & 3,14 & $-0,06$ & 0,31 & $-0,68$ & $-16,78$ & $-0,25$ & $-1,23$ & 1,30 \\
\hline
\end{tabular}

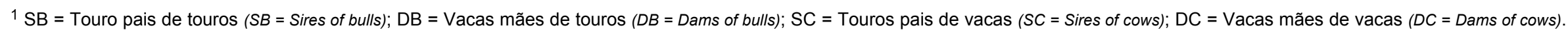


Tabela 3 - Estimativas de mudança genética anual (b) e erros-padrão (EP) para produção de leite, produção de gordura, duração da lactação e período seco, para cada trajetória de seleção

Table 3 - Estimates of annual genetic changes (b) and standard error (EP) for milk yield, fat yield, lactation length and days dry, for each path of selection

\begin{tabular}{|c|c|c|c|c|c|c|c|c|c|c|c|c|c|c|c|c|c|}
\hline \multirow{3}{*}{$\begin{array}{l}\text { Trajetória } \\
\text { de seleção } \\
\text { Selection } \\
\text { path }^{1}\end{array}$} & \multirow[b]{3}{*}{$\begin{array}{l}\text { Período }^{2} \\
\text { Period }^{2} \\
\end{array}$} & \multicolumn{4}{|c|}{$\begin{array}{l}\text { Leite } \\
\text { Milk }\end{array}$} & \multicolumn{4}{|c|}{$\begin{array}{l}\text { Gordura } \\
\quad \text { Fat }\end{array}$} & \multicolumn{4}{|c|}{$\begin{array}{l}\text { Duração da lactação } \\
\text { Lactation length }\end{array}$} & \multicolumn{4}{|c|}{$\begin{array}{l}\text { Período seco } \\
\text { Days dry }\end{array}$} \\
\hline & & \multicolumn{2}{|c|}{$\begin{array}{l}\text { Ponderado } \\
\text { Weighed }\end{array}$} & \multicolumn{2}{|c|}{$\begin{array}{l}\text { Não-ponderado } \\
\text { Unweighed }\end{array}$} & \multicolumn{2}{|c|}{$\begin{array}{l}\text { Ponderado } \\
\text { Weighed }\end{array}$} & \multicolumn{2}{|c|}{$\begin{array}{l}\text { Não-ponderado } \\
\text { Unweighed }\end{array}$} & \multicolumn{2}{|c|}{$\begin{array}{c}\text { Ponderado } \\
\text { Weighed }\end{array}$} & \multicolumn{2}{|c|}{$\begin{array}{l}\text { Não-Ponderado } \\
\text { Unweighed }\end{array}$} & \multicolumn{2}{|c|}{$\begin{array}{l}\text { Ponderado } \\
\text { Weighed }\end{array}$} & \multicolumn{2}{|c|}{$\begin{array}{c}\text { Não-ponderado } \\
\text { Unweighed }\end{array}$} \\
\hline & & $\mathrm{b}$ & EP & $\mathrm{b}$ & $\overline{\mathrm{EP}}$ & $\mathrm{b}$ & EP & $\mathrm{b}$ & EP & $\mathrm{b}$ & $\overline{\mathrm{EP}}$ & $\mathrm{b}$ & EP & $\mathrm{b}$ & $\overline{\mathrm{EP}}$ & $\mathrm{b}$ & $\mathrm{EP}$ \\
\hline \multirow{3}{*}{ SB } & 1 & 17,18 & 4,83 & 16,01 & 4,45 & 0,01 & 0,08 & 0,10 & 0,08 & 0,74 & 0,39 & 0,74 & 0,38 & $-1,56$ & 0,56 & $-1,30$ & 0,54 \\
\hline & 2 & 6,22 & 7,03 & 6,07 & 7,07 & $-0,02$ & 0,04 & $-0,02$ & 0,04 & 0,26 & 0,34 & 0,19 & 0,35 & $-0,52$ & 0,58 & $-0,30$ & 0,56 \\
\hline & 3 & 6,40 & 2,38 & 6,18 & 2,26 & 0,07 & 0,03 & 0,07 & 0,03 & 0,23 & 0,15 & 0,20 & 0,15 & $-0,35$ & 0,25 & $-0,33$ & 0,23 \\
\hline \multirow{3}{*}{ DB } & 1 & - & - & 5,00 & 5,81 & - & - & 0,01 & 0,11 & - & - & $-1,00$ & 0,71 & - & - & 0,68 & 0,66 \\
\hline & 2 & - & - & 7,94 & 7,83 & - & - & $-0,01$ & 0,04 & - & - & 0,82 & 0,76 & - & - & $-0,12$ & 0,14 \\
\hline & 3 & - & - & 13,21 & 2,65 & - & - & 0,03 & 0,04 & - & - & 0,42 & 0,31 & - & - & $-0,82$ & 0,30 \\
\hline \multirow{3}{*}{$\mathrm{SC}$} & 1 & 17,40 & 5,45 & 14,25 & 5,42 & 0,12 & 0,08 & 0,10 & 0,07 & 0,62 & 0,74 & 0,61 & 0,44 & $-1,13$ & 0,76 & $-0,87$ & 0,62 \\
\hline & 2 & 8,17 & 6,86 & 7,64 & 6,21 & $-0,01$ & 0,03 & 0,01 & 0,03 & 0,46 & 0,41 & 0,41 & 0,34 & $-0,42$ & 0,73 & $-0,63$ & 0,49 \\
\hline & 3 & 7,31 & 2,42 & 6,24 & 2,26 & 0,09 & 0,03 & 0,08 & 0,02 & 0,52 & 0,25 & 0,41 & 0,16 & $-0,38$ & 0,29 & $-0,37$ & 0,23 \\
\hline \multirow{3}{*}{$\mathrm{DC}$} & 1 & - & - & 0,77 & 2,80 & - & - & $-0,05$ & 0,04 & - & - & $-0,26$ & 0,35 & - & - & $-0,09$ & 0,25 \\
\hline & 2 & - & - & $-7,17$ & 2,28 & - & - & 0,03 & 0,01 & - & - & $-0,73$ & 0,40 & - & - & 0,03 & 0,25 \\
\hline & 3 & - & - & 4,69 & 1,19 & - & - & 0,04 & 0,01 & - & - & 0,20 & 0,14 & - & - & $-0,46$ & 0,10 \\
\hline
\end{tabular}

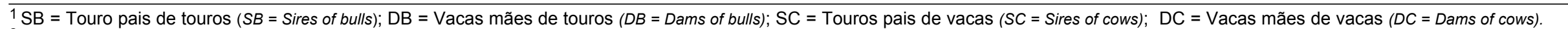
21 = 1952 a 1976; 2 = 1977 a 1997; 3 = Período total (1952 a 1997 - Whole period). 


\section{Tendência genética}

As tendências genéticas para produção de leite, produção de gordura, duração da lactação e período seco são apresentadas na Tabela 3 .

Para SB e SC, as tendências genéticas para produção de leite no primeiro período, de $17,18 \mathrm{~kg}$ e $17,40 \mathrm{~kg}$, respectivamente, foram maiores do que as do segundo e do período total. Nas raças Jersey e Holandesa, ganhos genéticos anuais da ordem de 232,04 e 80,9 kg de leite foram obtidos, respectivamente, por NIZAMANI e BERGER (1996) e ABDALLAHe McDANIEL (2000a), na trajetória de seleção de touros pais de touros.

Tendo em vista que a tendência genética para produção de leite foi, aproximadamente, 2,8 vezes maior no primeiro período do que no segundo, verifica-se que a seleção de reprodutores durante o programa de melhoramento foi menos eficiente. Provavelmente, a inclusão de caracteres relacionados com o tipo, como critério primário de seleção, foi determinante na obtenção desses resultados.

De modo geral, os ganhos obtidos para as demais características, em todas as trajetórias, foram inexpressivos, o que sugere que a seleção tenha sido direcionada somente para produção de leite.

A estimativa de tendência genética para a trajetória $\mathrm{DB}$, para produção de leite, foi maior no segundo período do que no primeiro, o que indica que a seleção de vacas mães de touros, durante o programa de melhoramento, tenha sido mais eficiente. Este fato pode ser observado ao se considerar o coeficiente de regressão associado a todo o período (1952 a 1997), pois o aumento dos valores genéticos de $\mathrm{DB}$, no segundo período, provocou maior inclinação da reta de regressão. Ganhos genéticos obtidos por meio de seleção de vacas foram relatados por ABDALLAH e McDANIEL (2000b), quando estes avaliaram produções de vacas da raça Holandesa, pertencentes a rebanhos leiteiros experimentais, nos Estados Unidos.

Em relação a DC, observa-se, no segundo período, tendência de diminuição dos valores genéticos para produção de leite, ao longo dos anos, em decorrência da diminuição do descarte e da seleção, uma vez que a maioria das vacas permanecia no rebanho.

Das quatro trajetórias de seleção, as estimativas de tendência genética para produção de leite, no primeiro período, foram maiores para as trajetórias $\mathrm{SB}$ e SC, enquanto no segundo período, isto é, durante o programa de melhoramento, os maiores ganhos foram obtidos nas trajetórias SC e DB. Durante todo o período, os maiores ganhos foram obtidos na trajetória DB. Os resultados encontrados neste estudo não são condizentes com os relatados por NIZAMANI e BERGER (1996) e BURNSIDE et al. (1992), que obtiveram maiores ganhos genéticos na trajetória $\mathrm{SB}$, o que permitiu aumentar o valor genético dos animais das raças avaliadas, com o passar dos anos.

Estimativas de ganho genético anual e respectivos erros-padrão para o ecótipo Mantiqueira, de cada trajetória de seleção, são apresentados na Tabela 4 .

Tabela 4 - Estimativas de ganho genético anual $(\Delta \mathrm{g})$ e erros-padrão $(E P)$ para produção de leite, produção de gordura, duração da lactação e período seco

Table 4 - Estimates of annual genetic gain $(\Delta g)$ and standard errors (EP) for milk yield, fat yield, lactation length and days dry, for each path of selection

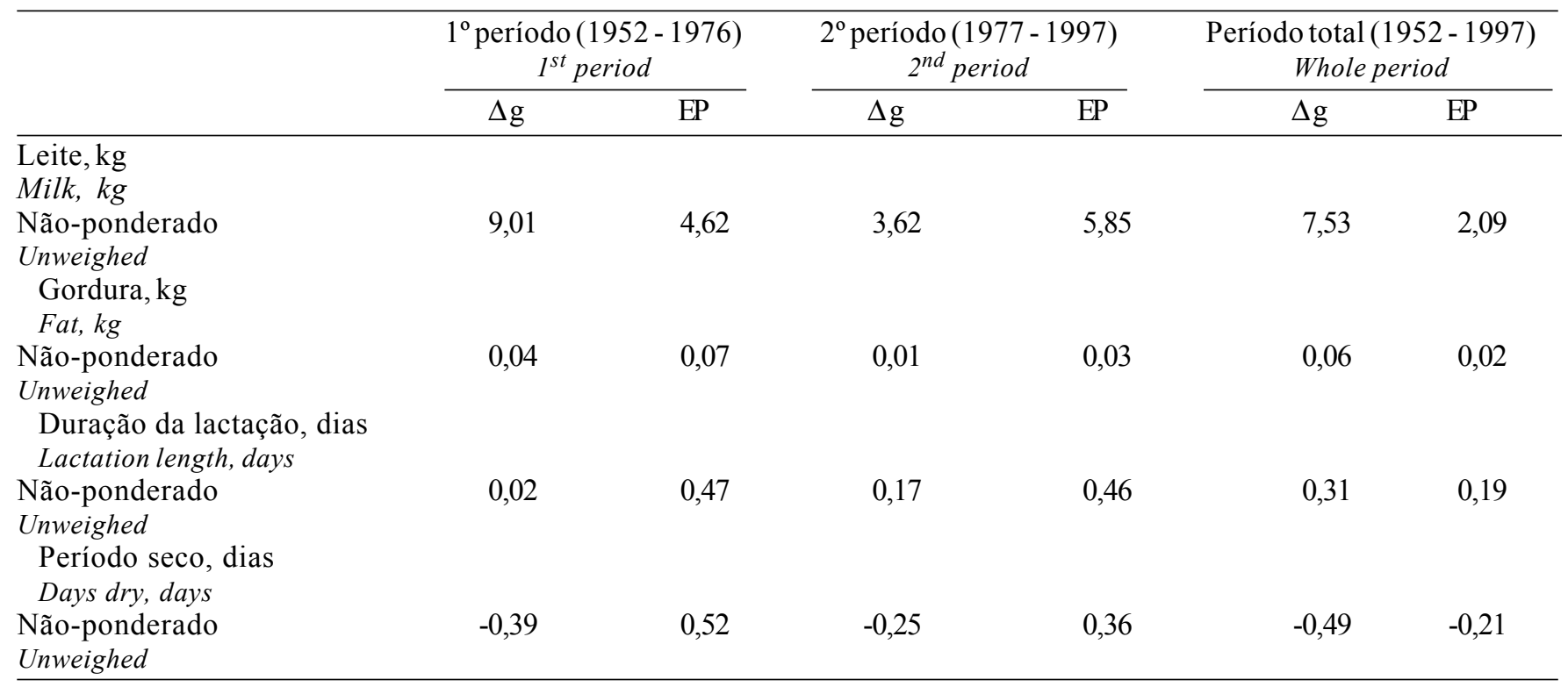


para produção de leite, para as quatro trajetórias, foram maiores no primeiro período (1952 a 1976). Em termos percentuais, o ganho genético foi da ordem de $0,5 \%$ e $0,2 \%$ ao ano, para produção de leite, no primeiro e no segundo período, respectivamente. Este resultado é inferior ao citado por RENDEL e ROBERTSON (1950), que relataram que, em um "rebanho fechado", a taxa provável de melhoramento por seleção é da ordem de $0,7 \%$ ao ano. Tal fato pode ser explicado pela seleção praticada para outras características como tipo, o que certamente reduziu o diferencial de seleção para produção de leite. Além disso, a não-utilização dos valores genéticos preditos para produção de leite, como critério de seleção dos touros, pode ter contribuído para o baixo progresso genético obtido. Para as demais características, os ganhos não foram expressivos.

\section{Conclusões}

Durante o programa de melhoramento os diferenciais de seleção das trajetórias $\mathrm{SB}$ e $\mathrm{SC}$, no primeiro período, mostraram maior eficiência na seleção de reprodutores, enquanto os diferenciais de seleção das trajetórias DB e DC mostraram maior eficiência na seleção de vacas.

As tendências genéticas obtidas para produção de gordura, duração da lactação e período de seco foram inexpressivas, o que sugere que a seleção tenha sido direcionada somente para produção de leite.

As taxas de ganho genético podem ser aumentadas, razão por que o programa de melhoramento de deve ser reestruturado, mediante a implementação de mudanças como seleção baseada somente em características de importância econômica, como produção de leite e de gordura, e utilização dos valores genéticos dos animais para estas características, como critério de seleção.

\section{Referências Bibliográficas}

ABDALLAH, J.M., McDANIEL, B.T. 2000a. Genetic change in milk, fat, days open, and body weight after calving based on three methods of sire selection. J. Dairy Sci., 83(6):1359-1363.

ABDALLAH, J.M., McDANIEL, B.T. 2000b. Genetic parameters and trends of milk, fat, days open, and body weight after calving in North Carolina experimental herds. J. Dairy Sci., 83(6):1364-1370.
BASTOS, J.F.P., LÔBO, R.B., OLIVEIRA, H.N. Estimativa da mudança genética na produção de leite em um rebanho da raça Pitangueiras. In: REUNIÃO ANUAL DA SOCIEDADE BRASILEIRA DE ZOOTECNIA, 35, 1998, Botucatu-SP. Anais... Botucatu: SBZ, 1998. p.338.

BOLDMAN, K.G., KRIESE, L.A., VAN VLECK, L.D. et al. 1995. A manual for use of MTDFREML. A set of programs to obtain estimates of variances and covariances. [DRAFT]. Beltsville: U.S. Department of Agriculture, Agricultural Research Service. $125 \mathrm{p}$.

BURNSIDE, E.B., JANSEN, G.B., CIVATI, G. et al. 1992. Observed and theoretical genetic trends in a large dairy population under intensive selection. J. Dairy Sci., 75(8):2242-2253.

GUARAGNA, G.P., GAMBINI, L.B., FIGUEIREDO, A.L. 1984. Mantiqueira: 30 Anos Depois. Nova Odessa: Instituto de Zootecnia, Secretaria de Agricultura e Abastecimento de São Paulo. 30p.

GUARAGNA, G.P., GAMBINI, L.B., FIGUEIREDO, A.L. et al. 1988. Eficiência reprodutiva do rebanho Mantiqueira da Estação Experimental de Zootecnia de Pindamonhangaba. I. Efeito de fatores de meio. Bol. Ind. Anim, 45(1):33-72.

NIZAMANI, A.H., BERGER, P.J. 1996. Estimates of genetic trend for yield traits of the registred Jersey population. J. Dairy Sci., 79(3):487-494.

RENDEL, J.M., ROBERTSON, A. 1950. Estimation of genetic gain in milk yield by selection in a closed herd of dairy cattle. J Genetics, 50(1):1-8.

ROMAN, R.M., WILCOX, C.J., LITTELL, R.C. 1999. Genetic Trends for milk yield of Jerseys and correlated changes in productive and reproductive performance. J. Dairy Sci., 82(1):196-204.

SANTOS, E.S., LEITE, P.R.M., LÔBO, R.B., DURÃES, M.C. 1990. Progresso genético do rebanho Gir leiteiro de Umbuzeiro, PB. Pesq. Agropec. Bras., 25(9):1339-1348.

VAN TASSELL, C.P., VAN VLECK, L.D. 1991. Estimates of genetic selection differentiais and generation intervals for four paths of selection. J. Dairy Sci., 74(3):1078-1086. 